\title{
Sacral Neuromodulation in Children
}

\author{
Andrew C. Strine, Alison C. Keenan, Shelly King, Benjamin M. Whittam*
}

Division of Pediatric Urology, Riley Hospital for Children at Indiana University Health, Indiana University School of Medicine, Indianapolis, Indiana, USA

\begin{abstract}
* Author for correspondence: Division of Pediatric Urology, Riley Hospital for Children at Indiana University Health, Indiana University School of Medicine, 705 Riley Hospital Drive, Suite 4230, Indianapolis, Indiana 46202, USA (telephone: 317-948-8556; fax: 317-274-7481; email: astrine@iupui.edu)
\end{abstract}

Key words: child; constipation; electrical stimulation therapy; fecal incontinence; urinary incontinence; urination disorders

This is the author's manuscript of the article published in final edited form as:

Strine, A. C., Keenan, A. C., King, S., \& Whittam, B. M. (2015). Sacral Neuromodulation in Children. Current Bladder Dysfunction Reports, 10(4), 332-337. http://doi.org/10.1007/s11884-015-0322-7 


\begin{abstract}
Sacral neuromodulation (SNM) has emerged as a treatment option for refractory lower urinary tract and bowel dysfunction in children. SNM is typically reserved for a select population of children who have failed an extended period of behavioral modification, biofeedback, and pharmacologic therapy. The surgical techniques for SNM in children are analogous to those in adults and include both one- and two-stage procedures as well as a peripheral nerve evaluation with the InterStim ${ }^{\circledR}$ system (Medtronic, Minneapolis, MN). Most studies have observed an improvement in symptoms and quality of life in children undergoing SNM for non-neurogenic and neurogenic lower urinary tract dysfunction but also a much higher reoperative rate than in adults. Further high-quality research is necessary to corroborate the results of earlier studies and to better define the indications for SNM in children.
\end{abstract}

\title{
Introduction
}

Sacral neuromodulation (SNM) has developed a growing interest for the treatment of refractory lower urinary tract dysfunction (LUTD) over the past 20 years. The Food and Drug Administration (FDA) initially approved the use of SNM with an implantable pulse generator (IPG) for the treatment of urgency urinary incontinence in 1997 followed by urgency-frequency syndrome and chronic non-obstructive urinary retention in 1999. Concurrent with its expanding indications in adults, SNM has emerged as a treatment option for refractory LUTD in children. Off-label use of SNM has been most thoroughly investigated in children with refractory dysfunctional voiding and also been reported in children with neurogenic LUTD and bowel dysfunction. In this review, we provide a critical evaluation of the literature and discuss the role of SNM in children with non-neurogenic and neurogenic LUTD. 


\section{Mechanism of Action}

The exact mechanism of action for SNM is not completely understood. Our current knowledge is founded on the neurophysiologic pathways for storage and emptying of the bladder in animal models. Proposed mechanisms of action are based on the selective excitation of somatic afferent fibers from the bladder, leading to the modulation of sacral and suprasacral reflexes that promote storage and emptying of the bladder. For overactive bladder, the excitation of these somatic afferent fibers is thought to suppress the micturition reflex at the sacral level and via the ascending pathways to the pontine micturition center. Inhibition at the sacral and supraspinal levels prevents the involuntary contraction of detrusor muscle, while still allowing for voluntary voiding due to selective avoidance of somatic afferent input from A $\delta$ fibers. Alternatively, the excitation of these somatic afferent fibers is thought to suppress the guarding reflex via the ascending pathways to the pontine micturition center and cortex for detrusor-external sphincter dyssynergia [1].

\section{Evaluation}

In addition to a thorough history and physical examination, a variety of diagnostic tools are available and should be considered for the evaluation of children with LUTD, including a bladder and bowel diary, validated questionnaires, urinalysis, renal and bladder ultrasound, and uroflowmetry with a post-void residual volume and with or without electromyography of perineal muscles. Urodynamic evaluation and additional imaging studies, such as a voiding cystourethrogram or spinal magnetic resonance imaging (MRI), should be considered in the evaluation of children with non-neurogenic LUTD, particularly when an invasive treatment like 
SNM is being considered [2, 3]. We routinely perform a videourodynamic evaluation and lumbosacral MRI on all children considered for SNM to characterize bladder dynamics and rule out correctable anatomic abnormalities (e.g. vesicoureteral reflux, bladder diverticula), occult neurogenic LUTD, and/or tethered spinal cord. MRI is contraindicated in children with an implanted device and therefore cannot be reserved for those who fail SNM.

\section{Initial Management}

For children with neurogenic LUTD, the storage of urine at a low intravesical pressure is essential for preservation of renal function and achievement of urinary continence. The mainstay of therapy includes early initiation of clean intermittent catheterization (CIC) and antimuscarinic therapy [6]. Off-label use of intravesical botulinum toxin A has proven to be effective and well tolerated in children with neurogenic LUTD, achieving urinary continence in $65-87 \%$ of children as well as reducing the mean maximum detrusor pressure to $<40 \mathrm{~cm} \mathrm{H}_{2} \mathrm{O}$ and increasing the compliance to $>20 \mathrm{~mL} / \mathrm{cm} \mathrm{H}_{2} \mathrm{O}$ in most studies [7]. Enterocystoplasty remains the gold standard for children with a low-capacity, poorly compliant, and/or overactive bladder, who fail a conservative approach. Children with reduced outflow resistance may also require a concomitant procedure at the bladder neck for achievement of urinary continence [6].

Overactive bladder is characterized by urinary urgency and often accompanied by frequency with or without incontinence, while dysfunctional voiding is characterized by the habitual contraction of the urethral sphincter and/or pelvic floor during voiding. Dysfunctional voiding manifests as urinary frequency, urgency, incomplete emptying, and/or recurrent urinary tract infections and may significantly impact quality of life (QOL). All of these conditions are idiopathic without identifiable underlying pathology [3]. Their initial management includes 
behavioral modification, initiation of a timed voiding regimen, and treatment of constipation. Biofeedback and/or pharmacologic therapy is typically offered as the next line of therapy. Antimuscarinic therapy has proven to be effective and well tolerated for the treatment of overactive bladder, and the off-label use of selective $\alpha$-adrenergic blockers has been promising for the treatment of dysfunctional voiding in several small, uncontrolled studies [4]. Off-label use of intravesical botulinum toxin A has also been investigated for the treatment of overactive bladder and detrusor-external sphincter dyssynergia in children. Preliminary results have been encouraging but require further investigation [5]. Several centers have developed a comprehensive program with a step-wise approach to the treatment of voiding dysfunction and achieved a success rate of $90 \%$ to $100 \%$ with behavioral modification, initiation of a regular voiding regimen, biofeedback, and/or pharmacologic therapy [4].

\section{Indications}

SNM is considered a last option for a select population of children with refractory LUTD due to its expense and invasive nature. Its off-label use has been investigated in children with overactive bladder, dysfunctional voiding, neurogenic LUTD, and bowel dysfunction. Currently, the indications for SNM have not been well defined in children [8].

Based on our experience and current literature, SNM should only be considered in children who have failed an extended period of behavioral modification, biofeedback, and pharmacologic therapy [9-15]. Several groups have also offered intravesical botulinum toxin A, transcutaneous electrical nerve stimulation, and percutaneous tibial nerve stimulation to this population of children but have not required them to fail these less invasive therapies prior to SNM $[13,15]$. 
There are no absolute contraindications to SNM. Several groups have excluded children with neurologic diseases or spinal deformities in their series [10,11, 14, 15]. Significant spinal deformities may complicate or even prohibit lead placement in the foramen of the third sacral spinal nerve (S3). Various implanted components of the InterStim ${ }^{\circledR}$ system may also be affected by or affect the following: cardiac devices, electrocautery, defibrillators, ultrasonic equipment, radiation therapy, magnetic resonance imaging, and theft detectors/screening devices [16].

Nearly all children require a two-stage procedure, allowing for a trial of variable duration (2-3 weeks) to determine whether they will benefit from IPG implantation. Certain groups have required an objective symptomatic improvement during the trial, whereas others have made a subjective determination or not reported their criteria to undergo IPG implantation in their series $[10,11,13-15]$. There are no established criteria for a successful trial. Dwyer et al. required at least a 50\% symptomatic improvement in a bladder and bowel diary when compared to a preoperative diary [15]. Alternatively, Groen et al. required at least a 50\% decrease in the episodes of urinary incontinence, post-void residual volume, need for CIC, and frequency in their series [13].

\section{Surgical Techniques}

The surgical techniques for SNM in children are analogous to those in adults. The InterStim ${ }^{\circledR}$ system (Medtronic, Minneapolis, MN) is the only commercially available implantable device for SNM. Both one- and two-stage procedures have been described in children $[9,15,17]$. The one-stage procedure involves the simultaneous implantation of the lead and IPG after eliciting a neurologic response to the stimulation of S3 (Table 1), while the two-stage procedure involves lead implantation and connection to an external pulse generator followed by staged IPG 
implantation after a successful trial. As an alternative to the first-stage procedure, a peripheral nerve evaluation (PNE) involves the implantation of a thin, flexible wire and connection to an external pulse generator under local anesthesia in an office-based setting. PNE may be feasible in older, more mature children but rarely is a viable option in the pediatric population.

The spinal foramena are often smaller and closer together in children than adults.

Furthermore, the lead excursion (distance of lead activity) is typically reduced due to the smaller size of nerves and nerve roots, making proper lead placement more technically challenging. A low threshold ( $<1 \mathrm{amp}$ ) is desired across all leads but may only be possible in up to 3 leads in smaller children. A tine may also be exposed in smaller children but will be buried once the lead is tunneled.

\section{Clinical Evidence - Efficacy}

\section{Non-neurogenic LUTD}

Several studies have demonstrated an improvement in symptoms and QOL in children undergoing SNM for non-neurogenic LUTD, but comparison is difficult due to heterogeneous cohorts and variable outcomes [10, 11, 13-15]. The most comprehensive series included 105 consecutive children with a median age of 8 years (range, 6-16), who underwent either a one- or two-stage procedure for refractory dysfunctional elimination syndrome (DES) with a median follow-up of 2.72 years (range, 0.01-9.63). A large proportion of children (42\%) previously underwent a bladder or urethral procedure in their series, including 9 children who failed intravesical injection of botulinum toxin A. Almost all children (94\%) experienced an improvement in at least 1 of the following symptoms after SNM: urinary incontinence, constipation, frequency and/or urgency, and nocturnal enuresis. Urinary incontinence, 
constipation, frequency and/or urgency, and nocturnal enuresis improved in 89 of 101 (88\%), 73 of 92 (79\%), 54 of 81 (67\%), and 59 of 89 (66\%) of children, respectively, and resolved in 41 of 101 (41\%), 37 of 92 (40\%), 23 of 81 (28\%), and 25 of 89 (28\%) children undergoing SNM, respectively [15]. Groen et al. observed a similar response rate in a retrospective study of 18 consecutive children with a mean age of 15 years (range, 9-17), who underwent a two-stage procedure for refractory overactive bladder, DES, Fowler syndrome, and neurogenic bladder with a median follow-up of 11 months (range, 0.5-180.0). A long-term full response (defined as $100 \%$ satisfaction and greater than $90 \%$ objective improvement in at least 1 of the following criteria for implantation: urinary incontinence, elevated postvoid residual volume, need to perform CIC, and frequency) was achieved in 6 of 15 (40\%) children, whereas a long-term partial response (defined as satisfaction and 50-90\% objective improvement in at least 1 criteria for implantation) was achieved in 5 of 15 (33\%) children for a total response rate of $73 \%$ after SNM. Children with urinary incontinence also experienced a significant decrease in the mean number of incontinent episodes (23.2 to 1.3), and children undergoing CIC experienced a significant decrease in the mean frequency of catheterization per day after SNM (5.2 to 2.0) [13]. These studies largely relied on non-validated questionnaires or patient-reported bladder and bowel diaries, which are subjective and have inherent limitations [18].

In an effort to provide more objective evidence of improvement in both symptoms and QOL after SNM, Stephany et al. evaluated the health-related QOL of 14 children with a median age of 10 years (range, 5-17), who underwent a two-stage procedure for refractory LUTD with a median follow-up of 6 months (range, 0.5-15.0). Health-related QOL was evaluated before and after SNM, using the validated Vancouver non-neurogenic LUTD/DES questionnaire and PedsQL 4.0 Generic Core Scale. Median Vancouver non-neurogenic LUTD/DES scores 
significantly improved after SNM (23.0/52 to 10.5/52), and mean total scores for PedsQL 4.0

Generic Core Scale (75.0/100 to 84.0/100) and subscores for psychosocial (70.6/100 to 81.4/100) but not physical (85.9/100 to 86.9/100) QOL significantly improved after SNM. These improvements remained durable over limited follow-up. Nighttime and daytime urinary incontinence, urgency, and fecal incontinence also significantly improved after SNM [14].

No studies were randomized or included a matched control group. Results may therefore be attributed to the placebo effect or maturation of their cohorts [10, 11, 13-15]. Dwyer et al. demonstrated that urinary frequency and/or urgency resolved in a significantly greater proportion of children who had a follow-up of at least 2.72 years (38\% vs. 14\%), although none of the other symptoms correlated with the duration of follow-up [15]. Furthermore, the generalizability of these studies is limited due to the heterogeneity of their cohorts. Most children were labeled as having an exclusionary diagnosis of DES rather than subgrouped, according to the terminology from the Standardization Committee of the International Children's Continence Society [10, 11, 14, 15]. In the series by Groen et al., the subgrouping of LUTD allowed for a more meaningful determination of who would benefit from SNM. Children with overactive bladder, DES, and Fowler syndrome seemed to experience an improvement after SNM, while those with neurogenic bladder did not [13].

\section{Neurogenic LUTD}

Off-label use of SNM is more controversial in children with neurogenic LUTD. Several European studies have investigated its use in this population with encouraging results [9, 12].

Guys et al. performed a prospective randomized controlled trial of 42 children with urinary incontinence due to neurogenic LUTD, who underwent a one-stage procedure and were followed 
for a minimum of 12 months. Most children (79\%) had an underlying etiology of spina bifida. Inclusion criteria were at least 2 of the following: duration of continence less than or equal to 90 minutes, postvoid residual volume greater than $50 \%$ of functional bladder capacity, compliance less than $15 \mathrm{~mL} / \mathrm{cm} \mathrm{H}_{2} \mathrm{O}$, intravesical or leak point pressure greater than $40 \mathrm{~cm} \mathrm{H}_{2} \mathrm{O}$, and detrusor overactivity with peak pressures greater than $40 \mathrm{~cm} \mathrm{H}_{2} \mathrm{O}$. Those randomized to the control group were treated with antimuscarinic therapy and bulking agents for urinary incontinence and vesicoureteral reflux as needed. A subjective improvement was observed in several children undergoing SNM, including improved bowel function in 9 children, resolution of urinary tract infections in 5 children, and improved bladder sensation in 6 children. One child experienced a resolution of urinary incontinence but still required CIC after SNM. A comparison of urodynamic parameters also identified a significantly increased leak point pressure but a significantly decreased functional bladder capacity in children undergoing SNM. For children undergoing SNM, the compliance and functional bladder capacity were significantly improved on urodynamic evaluation at 6 and 9 but not 12 months when compared to baseline. Furthermore, the total bladder capacity was increased on all urodynamic evaluations. An improvement in detrusor stability was also achieved in 30\% of children with detrusor overactivity. The authors suggested that the neurologic lesions of children in their series were too severe to demonstrate a significant improvement after SNM [9].

Based on these promising results, Haddad et al. performed a prospective randomized controlled trial of 33 children undergoing SNM for neurogenic urinary and/or fecal incontinence at multiple centers in France. All children underwent a one-stage procedure and were randomized to either the activation or deactivation of their device for 6 months and subsequently crossed over to the other group for 6 months with a washout period of 45 days. Thirty percent of 
children had an underlying etiology of spina bifida with similar inclusion criteria to the prior study. A response (defined as a resolution or greater than 50\% improvement in the number of urinary and/or fecal incontinent episodes with a minimal need for protection) was achieved in 17 of 21 (81\%) children with urinary incontinence and 14 of 18 children (78\%) with fecal incontinence. Five children experienced a continued response after deactivation of their device, suggesting a permanent remodeling of neurophysiologic pathways [12]. Roth et al. reported a similar phenomenon in 2 children undergoing SNM for refractory DES [11]. Based on a crossover analysis, SNM was significantly more effective than conservative therapy for the treatment of both urinary and fecal incontinence. A comparison of urodynamic parameters also identified a significantly increased cystometric bladder capacity (+24.27 mL vs. $-37.45 \mathrm{~mL})$ but a significantly greater amount of detrusor overactivity (+1 vs. -0.36) in children with SNM [12]. A consistent improvement in urodynamic parameters was not observed in children with SNM, as in the prior study $[9,12]$. The authors suggested that a subjective improvement in symptoms may precede an objective improvement in urodynamic parameters [12]. Others have argued that the routine use of urodynamic evaluation may be unwarranted due to lack of correlation with symptomatology [18]. Stephany et al. recommended the limited use of urodynamic evaluation in children with non-neurogenic LUTD who have a poor response to SNM due to its invasive nature [14].

The quality of these studies is superior to the evidence for children with non-neurogenic LUTD, given their randomization and inclusion of a control group or crossover design. However, the generalizability of these studies is still limited by their heterogeneous cohorts, which included a variety of neurologic diseases as well as congenital anorectal and urologic abnormalities [9, 12]. Nevertheless, SNM proved to be feasible and effective in children with 
neurogenic LUTD. Neurologic diseases or spinal deformities have been considered a contraindication to SNM due to perceived difficulty with accessing the S3 foramen and presumed paucity of existing neurotransmission. Haddad et al. were successfully able to place a lead in the S3 foramen in 33 of 41 children (81\%) in their series and achieve a similar response rate to children with non-neurogenic LUTD [10-13, 15].

\section{Bowel dysfunction}

While the use of SNM has been thoroughly investigated in adults with constipation and fecal incontinence, a paucity of evidence exists in children. Bladder and bowel dysfunction should be evaluated concomitantly due to their close relationship and frequent co-occurrence in children [3]. Several studies have evaluated for improvement in bowel function in children undergoing SNM for refractory LUTD [9-12, 14, 15]. Dwyer et al. noted that most children (88\%) suffered from both bladder and bowel dysfunction in their series and demonstrated an improvement in constipation in 79\% of children undergoing SNM [15]. Stephany et al. also observed a significant improvement in fecal incontinence in 5 children undergoing SNM in their series [14]. Haddad et al. achieved a similar response rate of $71 \%$ for children with neurogenic urinary and/or fecal incontinence undergoing SNM. Incontinence was mixed in 19 of 33 (58\%) children and fecal only in 5 of 33 (15\%) children in their series, but a consistent improvement in rectomanometric parameters was not demonstrated in children with SNM [12]. Only 1 recent study has primarily focused on the use of SNM in children with bowel dysfunction. This series included 13 girls with a median age of 15.2 years (range, 10-18), who underwent a two-stage procedure for refractory functional constipation and were followed for a minimum of 6 months. Most children (92\%) experienced an improvement in constipation after SNM with a significant 
improvement in the Average Cleveland Clinic constipation score (20.9/30 to 8.4/30), frequency of defecation, abdominal pain, straining, and sensation of incomplete evacuation. Absenteeism from school completely resolved after SNM, compared to an incidence of 77\% preoperatively. Despite its retrospective design and the small size of its cohort, the preliminary results of this study are encouraging but require further investigation [19].

\section{Clinical Evidence - Complications}

Complications rates in children undergoing SNM have ranged from $14 \%$ to $56 \%$ with the most common complications including: pain at the site of implantation ( $0-15 \%)$, surgical site infection (SSI) (0 - 12\%), lead migration or fracture ( $0-10 \%)$, IPG malfunction or its connection $(0-9 \%)$, undesired sensation $(0-8 \%)$, and seroma $(0-4 \%)[9-15,19]$.

A majority of complications are managed with observation or device reprogramming in adults. However, a much higher reoperative rate for revision, replacement, or explantation of the device (13 - 56\%) has been observed in children undergoing SNM [9-15, 19]. In the study with the longest duration of follow-up, 85 reoperations were performed in 59 of 105 (56\%) children for an average of 0.79 additional unplanned operations per child. A total of 47 revisions or replacements were performed in 36 children at a median time of 1.26 years from their initial procedure (range, $0.01-6.25$ years), and 38 explantations (22 due to resolution or stable improvement in symptoms) were performed in 36 children at a median time of 2.36 years (range, $0.03-9.05$ years) [15].

Several technical considerations may decrease the risk of complications and reoperation in children. The most recent model of IPG (InterStim ${ }^{\circledR}$ II neurostimulator, model 3058) is 37\% smaller by volume and $50 \%$ lighter than the prior model as well as directly connects to the lead, 
eliminating the need for a quadripolar extension. These modifications are important in children, who have less subcutaneous tissue in their gluteal region than adults. The newer leads also consist of tines that are deployed for fixation rather than being anchored to the periosteum. This modification may minimize lead migration during the trial, pain at the implantation site, and difficulty with revision or explantation [16]. Several authors have suggested a need to further miniaturize and redesign the device for children $[12,15,18]$. Another technical consideration is the decision to perform a one- or two-stage procedure. A one-stage procedure prevents the accidental migration or removal of the lead during the trial and eliminates the need for a second anesthetic. Dwyer et al. demonstrated a significantly decreased rate of explantation but no difference in the rate of SSI in children undergoing a one-stage procedure, although only a small number of children (15\%) elected for this approach in their series [15].

Lead migration or fracture due to somatic growth is unique to children. Clark et al. observed an association between lead malfunction and somatic growth in 4 children undergoing SNM at a mean age of 12.1 years (range, 11-13.2). Three children (75\%) required a total of 5 revisions due to lead malfunction with a mean time of 1.5 years (range, $0.7-2.8$ years) and somatic growth of $8.1 \mathrm{~cm}$ (range, $4-12.5 \mathrm{~cm}$ ) between revisions. Efficacy was restored after replacing the lead in a similar position during all revisions. Their reoperative rate was higher than other series, which was attributed to an increased pubertal growth due to the older age of children and absence of comorbidities in their cohort [20].

\section{Conclusions}

A growing body of evidence supports the off-label use of SNM in children with refractory LUTD and bowel dysfunction. Most studies have demonstrated an improvement in symptoms 
and QOL in children undergoing SNM for non-neurogenic and neurogenic LUTD but also a much higher reoperative rate than in adults $[9-15,19]$. Comparison is difficult due to different designs, heterogeneous cohorts, and variable outcomes. Higher quality evidence is needed, as most studies are retrospective and lack a randomized or matched control group.

The use of SNM will continue to expand in children with refractory LUTD due to the challenging nature of treating this population. Further research is necessary to corroborate the results of earlier studies and to better define the indications for SNM in children [8].

Forthcoming studies should subgroup cohorts according to the terminology from the Standardization Committee of the International Children’s Continence Society and include a randomized or matched control group. Researchers should strive to establish a uniform criterion for the definition of outcomes, using a variety of validated questionnaires and objective clinical data $[3,18]$. Further research should also focus on the neurophysiologic pathways that promote the storage and emptying of the bladder, as this will facilitate the development of novel treatment options for LUTD in children.

\section{Conflict of Interest}

No authors have any conflicts of interest to disclose.

\section{Human Rights, Welfare of Animals, and Informed Consent}

This article does not contain any studies with human or animal subjects performed by any of the authors. 
Table 1. Neurologic response to stimulation of sacral spinal nerves

\begin{tabular}{|l|l|l|l|}
\hline Sacral spinal nerve & Motor - pelvic floor & \multicolumn{1}{|c|}{$\begin{array}{c}\text { Motor - lower } \\
\text { extremity }\end{array}$} & Sensory \\
\hline S2 & $\begin{array}{l}\text { Anterior-posterior } \\
\text { shortening of } \\
\text { perineum (clamp), } \\
\text { deepening and } \\
\text { flattening of } \\
\text { intergluteal fold } \\
\text { (bellows) }\end{array}$ & $\begin{array}{l}\text { Rotation of hip, } \\
\text { contraction of calf, } \\
\text { rotation of heel, } \\
\text { dorsiflexion of foot }\end{array}$ & Genital \\
\hline S3 & Bellows & $\begin{array}{l}\text { Dorsiflexion of great } \\
\text { toe }\end{array}$ & $\begin{array}{l}\text { Genital, perineal, and } \\
\text { anal }\end{array}$ \\
\hline S4 & Bellows & None & Anal \\
\hline
\end{tabular}

\section{References}

Recently published references of particular interest have been highlighted as:

- Of importance

•• Of major importance

1. Zhang F, Zhao S, Shen B, Wang J, Nelson DE, Roppolo JR, et al. Neural pathways involved in sacral neuromodulation of reflex bladder activity in cats. Am J Physiol Renal Physiol. 2013;304:F710-7.

2. Hoebeke P, Bower W, Combs A, de Jong T, Yang S. Diagnostic evaluation of children with daytime incontinence. J Urol. 2010;183:699-703.

3. Austin PF, Bauer SB, Bower W, Chase J, Franco I, Hoebeke P, et al. The Standardization of Terminology of Lower Urinary Tract Function in Children and Adolescents: Update Report from the Standardization Committee of the International Children's Continence Society. J Urol. 2014;191:1863-65.e13. 
4. Chase J, Austin P, Hoebeke P, McKenna P. The management of dysfunctional voiding in children: a report from the Standardisation Committee of the International Children's Continence Society. J Urol. 2010;183:1296-302.

5. Hassouna T, Gleason JM, Lorenzo AJ. Botulinum toxin A's expanding role in the management of pediatric lower urinary tract dysfunction. Curr Urol Rep. 2014;15:426.

6. Rawashdeh YF, Austin P, Siggaard C, Bauer SB, Franco I, de Jong TP, et al. International Children's Continence Society's recommendations for therapeutic intervention in congenital neuropathic bladder and bowel dysfunction in children. Neurourol Urodyn. 2012;31:615-20.

7. Gamé X, Mouracade P, Chartier-Kastler E, Viehweger E, Moog R, Amarenco G, et al. Botulinum toxin-A (Botox) intradetrusor injections in children with neurogenic detrusor overactivity/neurogenic overactive bladder: a systematic literature review. J Pediatr Urol. 2009;5:156-64.

8. De Gennaro M, Capitanucci ML, Mosiello G, Zaccara A. Current state of nerve stimulation technique for lower urinary tract dysfunction in children. J Urol. 2011;185:1571-7.

9. - Guys JM, Haddad M, Planche D, Torre M, Louis-Borrione C, Breaud J. Sacral neuromodulation for neurogenic bladder dysfunction in children. J Urol. 2004;172:1673-6. This is only 1 of 2 prospective randomized controlled trials, investigating the use of SNM in children with neurogenic LUTD.

10. Humphreys MR, Vandersteen DR, Slezak JM, Hollatz P, Smith CA, Smith JE, et al. Preliminary results of sacral neuromodulation in 23 children. J Urol. 2006;176:2227-31.

11. Roth TJ, Vandersteen DR, Hollatz P, Inman BA, Reinberg YE. Sacral neuromodulation for the dysfunctional elimination syndrome: a single center experience with 20 children. J Urol. 2008;180:306-11. 
12. • Haddad M, Besson R, Aubert D, Ravasse P, Lemelle J, El Ghoneimi A, et al. Sacral neuromodulation in children with urinary and fecal incontinence: a multicenter, open label, randomized, crossover study. J Urol. 2010;184:696-701. This is only 1 of 2 prospective randomized controlled trials, investigating the use of SNM in children with neurogenic LUTD.

13. Groen LA, Hoebeke P, Loret N, Van Praet C, Van Laecke E, Ann R, et al. Sacral neuromodulation with an implantable pulse generator in children with lower urinary tract symptoms: 15-year experience. J Urol. 2012;188:1313-7.

14. Stephany HA, Juliano TM, Clayton DB, Tanaka ST, Thomas JC, Adams MC, et al. Prospective evaluation of sacral nerve modulation in children with validated questionnaires. J Urol. 2013;190:1516-22.

15. • Dwyer ME, Vandersteen DR, Hollatz P, Reinberg YE. Sacral neuromodulation for the dysfunctional elimination syndrome: a 10-year single-center experience with 105 consecutive children. Urology. 2014;84:911-7. This is the largest series of children undergoing SNM due to LUTD.

16. Medtronic Sacral Neuromodulation System Information for Prescribers. Medtronic, Inc. 2012. http://professional.medtronic.com/pt/uro/snm/prod/index.htm. Accessed 31 Jan 2015.

17. McGee SM, Routh JC, Granberg CF, Roth TJ, Hollatz P, Vandersteen DR, et al. Sacral neuromodulation in children with dysfunctional elimination syndrome: description of incisionless first stage and second stage without fluoroscopy. Urology. 2009;73:641-4.

18. Fox JA, Reinberg YE. Incontinence. Pediatric sacral neuromodulation for refractory incontinence. Nat Rev Urol. 2010;7:482-3. 
19. van Wunnik BP, Peeters B, Govaert B, Nieman FH, Benninga MA, Baeten CG. Sacral neuromodulation therapy: a promising treatment for adolescents with refractory functional constipation. Dis Colon Rectum. 2012;55:278-85.

20. Clark C, Ngo T, Comiter CV, Anderson R, Kennedy W. Sacral nerve stimulator revision due to somatic growth. J Urol. 2001;186:1576-80. 